Check for updates

Cite this: Nanoscale Adv., 2019, 1, 1124

\title{
An in situ Bi-decorated BiOBr photocatalyst for synchronously treating multiple antibiotics in water $\dagger$
}

\author{
Feng Cao, (D) a Jianmin Wang, ${ }^{a}$ Yunan Wang, ${ }^{a}$ Jun Zhou, ${ }^{a}$ Song Li, (D) a Gaowu Qin (D) *a \\ and Weiqiang Fan ${ }^{\star b}$
}

Currently, there is an urgent demand for developing new materials to remove antibiotics in the water environment, especially for the simultaneous degradation of multiple antibiotics. Here, we fabricated a novel $\mathrm{Bi} / \mathrm{BiOBr}$ heterostructure via an in situ solvothermal strategy, and it exhibited excellent visiblelight-responsive photocatalytic activity for synchronously removing multiple antibiotics coexisting in water. The Bi nanoparticles could extend the light absorption spectra of the sample and further facilitate electron-hole pair separation. The in-depth electron spin resonance (ESR) results confirm that the active species in $\mathrm{Bi} / \mathrm{BiOBr}$ are holes $\left(\mathrm{h}^{+}\right)$and superoxide radicals $\left(\cdot \mathrm{O}_{2}^{-}\right)$under irradiation, and it is also proved that $\mathrm{Bi}$ could selectively reduce the formation of $\cdot \mathrm{O}_{2}{ }^{-}$in the $\mathrm{BiOBr}$ matrix. The coexisting system of TC (tetracycline hydrochloride), CIP (ciprofloxacin) and DOX (doxycycline) could be simultaneously photodegraded to approximately $0 \%$ within $30 \mathrm{~min}$ by the $\mathrm{Bi} / \mathrm{BiOBr}$ photocatalyst.

Received 6th September 2018 Accepted 11th December 2018

DOI: 10.1039/c8na00197a

rsc.li/nanoscale-advances
Bismuth oxyhalides (BiOX, $\mathrm{X}=\mathrm{Br}$ and I) as typical layered tetragonal semiconductors have been widely applied in the fabrication of visible-light-responsive photocatalysts. ${ }^{6,7}$ However, the photocatalytic performance of bare BiOX (X $=\mathrm{Br}$ and I) is still limited because of its high electron-hole recombination rate and poor surface adsorptive ability. Combining $\operatorname{BiOX}(\mathrm{X}=\mathrm{Br}$ and $\mathrm{I}$ ) with noble metals to obtain uniform heterostructures is an effective strategy to improve the photocatalytic activity. For instance, Yu et al. demonstrated that different noble metal particles (Ag, Pt, Pd, etc.) could largely improve photocatalytic activities of $\mathrm{BiOX}(\mathrm{X}=\mathrm{Br}$ and $\mathrm{I})$, which could be attributed to the strong visible light absorption and low recombination rate of the $\mathrm{e}^{-} / \mathrm{h}^{+}$pairs caused by the presence of noble metal particles. ${ }^{8-10}$ However, the introduction of noble metals generates the unavoidable problem of high production costs, and so, replacing noble metals with transition metals will be key to designing photocatalysts with the $\mathrm{BiOBr}-$ metal form. Moreover, metallic Bi has been verified to be able to largely improve the photodegradation efficiency of $\mathrm{BiOX}$ (X $=$ $\mathrm{Cl}, \mathrm{Br}$ and I) for various dyes through in situ synthesis in many studies, which can effectively decrease the recombination rate of electron-hole pairs and improve their light adsorption as a result of surface plasmon resonance (SPR). ${ }^{11-18}$

In this work, we fabricated a novel visible-light-responsive photocatalyst $(\mathrm{Bi} / \mathrm{BiOBr})$, in which $\mathrm{Bi}$ nanoparticles were in situ-embedded in the BiOBr hierarchical structure with diethylene glycol (DEG) as the reaction medium. Compared to previous studies, the present preparation method relies on in situ reduction and growth of Bi nanoparticles on the surface of BiOBr, which can suppress the agglomeration of $0 \mathrm{D}$ 
nanoparticles to expose more active sites. The visible-light photodegradation ability of the $\mathrm{Bi} / \mathrm{BiOBr}$ nanomaterial was indepth studied by degrading two-component/three-component mixed antibiotics. The effect of the heterostructure on the photocatalytic performance of $\mathrm{Bi} / \mathrm{BiOBr}$ hierarchical microflowers has been discussed compared to that of pure $\mathrm{BiOBr}$. Meanwhile, the $\mathrm{Bi} / \mathrm{BiOBr}$ sample is very air-stable, and its activity remains effective after cyclic experimental tests.

\section{Experimental}

\section{Preparation of samples}

Typically, appropriate amounts of $\mathrm{Bi}\left(\mathrm{NO}_{3}\right)_{3} \cdot 4 \mathrm{H}_{2} \mathrm{O}$ and PVP (polyvinylpyrrolidone) were mixed into $10 \mathrm{~mL}$ of DEG $\left(\mathrm{HOCH}_{2} \mathrm{CH}_{2} \mathrm{OCH}_{2} \mathrm{CH}_{2} \mathrm{OH}\right)$ under magnetic stirring. Then, the mixture was transferred into DEG solution $(10 \mathrm{~mL})$ containing $\mathrm{KBr}$. After continuous stirring for 20 minutes, the solution mixture was transferred and sealed in a Teflon-lined autoclave $(25 \mathrm{~mL})$. It was kept for $12 \mathrm{~h}$ at $180^{\circ} \mathrm{C}$ and then cooled naturally. A precipitate was obtained after separation, washing and drying. Meanwhile, bare BiOBr powder was synthesized via the same method without DEG.

\section{Characterization of samples}

$\mathrm{X}$-ray diffraction (XRD) was performed using a Rigaku-D/max $2500 \mathrm{~V}$ using $\mathrm{Cu} \mathrm{K} \alpha$ radiation. X-ray photoelectron spectroscopy (XPS) was performed using a Thermo instrument using Al $\mathrm{K} \alpha$ radiation. Scanning electron microscopy (SEM) was performed using a JEOL JEM-7100F. TEM was performed using a JEOL JEM-200CT at $200 \mathrm{kV}$ accelerating voltage. The optical ability was examined using a UV-visible spectrophotometer (PerkinElmer Lambda 750S). ESR was measured using a Bruker model A300-10/12 spectrometer.

\section{Photocatalytic activity studies}

Photocatalytic reactions were performed in a Pyrex reactor using a $300 \mathrm{~W}$ Perfect xenon lamp with or without a UV-cut filter $(400$ $\mathrm{nm}) .60 \mathrm{mg}$ of photocatalyst was poured into $75 \mathrm{~mL}$ fresh pollutant. Prior to irradiation, they were stirred for 60 minutes to ensure that the adsorption equilibrium had been reached. During the photoreactions, $4 \mathrm{~mL}$ of reaction solution were taken out at given time intervals for chemical analysis. The dye concentration was analysed by UV-vis absorption spectroscopy. The antibiotic concentration was determined using a Waters e2695 high-performance liquid chromatograph (HPLC). The wavelengths of the UV detector were set at $265 \mathrm{~nm}$ (DOX), $280 \mathrm{~nm}$ (CIP) and $278 \mathrm{~nm}$ (TC).

\section{Results and discussion}

The phase purity and composition were first measured by XRD characterization. As shown in Fig. 1, the observed reflection peaks at $2 \theta=25.16,32.20,46.20,57.12$ and $67.40^{\circ}$ correspond to the planes of (101), (110), (200), (212) and (220), in order, which can be readily indexed to the tetragonal phase of $\mathrm{BiOBr}$ [space group: P4/nmm (129)] (corresponding JCPDS File no. 09-

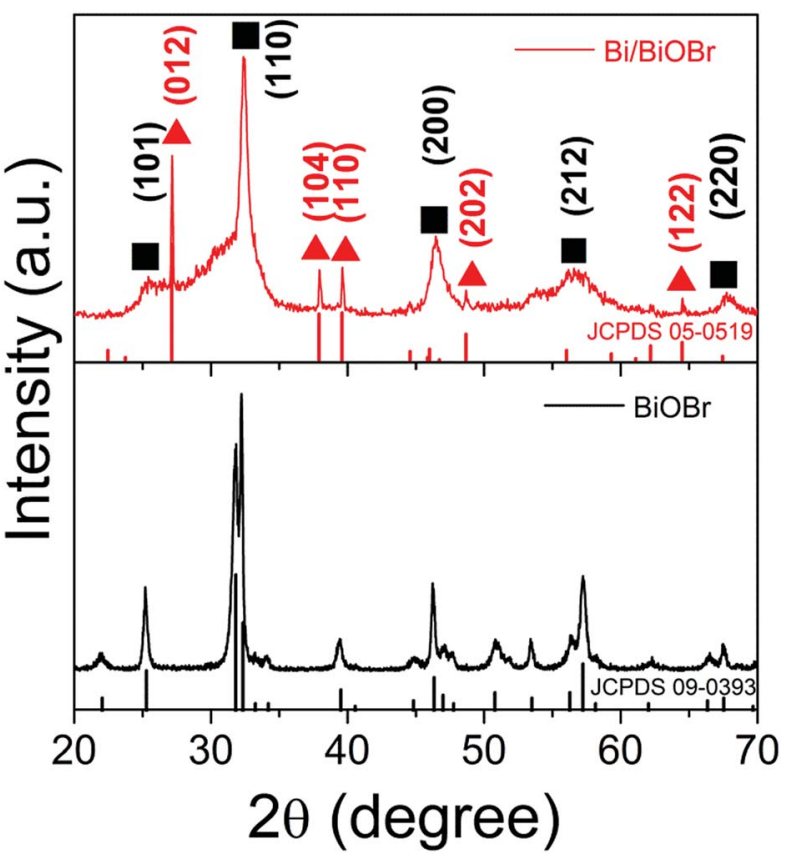

Fig. 1 XRD patterns of the $\mathrm{Bi} / \mathrm{BiOBr}$ and $\mathrm{BiOBr}$ samples.

0393). At the same time, the diffraction peaks at $2 \theta=27.17$, $38.27,39.67,48.93$ and $64.68^{\circ}$ correspond to the planes of $(012)$, (104), (110), (202) and (122), in order, which can be readily indexed to metallic Bi (JCPDS 05-0519). No impurities can be detected from this pattern, which confirms the successful fabrication of the $\mathrm{Bi} / \mathrm{BiOBr}$ heterostructure. For comparison, bare tetragonal BiOBr (JCPDS 09-0393) was also prepared by the same method without adding the reducer DEG solvent.

The SEM image of the $\mathrm{Bi} / \mathrm{BiOBr}$ nanoflowers in Fig. 2a shows that they have a uniform flowerlike morphology. The particle size was in the range of $1-3 \mu \mathrm{m}$. Fig. $2 \mathrm{~b}$ further reveals that the microflowers consist of overlapping nanoplatelets and their

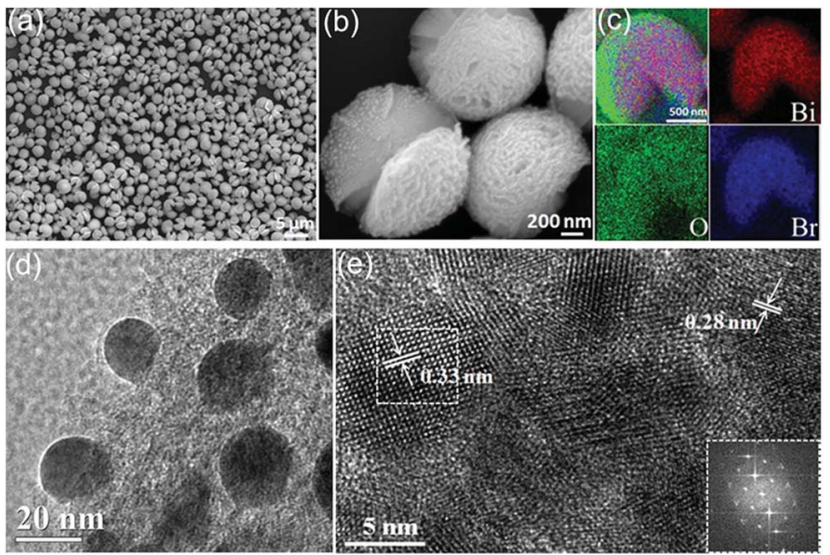

Fig. 2 (a) Low-magnification FESEM image of the $\mathrm{Bi} / \mathrm{BiOBr}$ nanoflowers. (b) Enlarged FESEM image. (c) The corresponding elemental mapping images of $\mathrm{Bi}, \mathrm{O}$ and $\mathrm{Br}$ elements; (d) representative TEM image; and (e) HRTEM image and FFT pattern (inset) of the edge area of the $\mathrm{Bi} / \mathrm{BiOBr}$ nanoflowers. 
thickness is approximately $25-35 \mathrm{~nm}$. The corresponding XEDS energy mapping images of the microflowers in Fig. 2c firmly identify their composition, in which $\mathrm{Bi}, \mathrm{O}$ and $\mathrm{Br}$ have been detected. Furthermore, the SEM image of the BiOBr sample displays a nanoflake morphology (Fig. S1†). The TEM image in Fig. 2d further shows that many Bi nanoparticles with approximately $15 \mathrm{~nm}$ diameter were evenly attached to the $\mathrm{BiOBr}$ surface. Two types of lattice fringes were observed in the HRTEM image (Fig. 2e), and they correspond to the (102) planes of BiOBr and (012) planes of Bi, respectively.

XPS measurements were also performed to investigate the chemical composition and elemental valence states. ${ }^{19}$ The survey XPS result (Fig. 3a) supports the presence of $\mathrm{O}, \mathrm{Br}$ and Bi. The Bi $4 \mathrm{f}$ XPS spectrum is depicted in Fig. $3 \mathrm{~b}$, and it can be split into two parts, $\mathrm{Bi} 4 \mathrm{f} 7 / 2$ and $\mathrm{Bi} 4 \mathrm{f} 5 / 2$, and the shake-up satellite peak at $155.7 \mathrm{eV}$ was ascribed to metallic $\mathrm{Bi}^{20,21}$ In the $\mathrm{O} 1 \mathrm{~s}$ spectra of Fig. 3c, the peaks at $529.9 \mathrm{eV}$ and $531.5 \mathrm{eV}$ can be ascribed to $\mathrm{Bi}-\mathrm{O}$ bonds and $\mathrm{Br}-\mathrm{O}$ bonds in $\mathrm{BiOBr}^{22,23}$ The peaks at $533.0 \mathrm{eV}$ were caused by the surface adsorbed $\mathrm{OH}$ group. ${ }^{24} \mathrm{In}$ the $\mathrm{Br} 3 \mathrm{~d}$ spectra (Fig. 3d), the binding energies of $67.5 \mathrm{eV}$ and $68.5 \mathrm{eV}$ were attributed to $\mathrm{Br} 3 \mathrm{~d} 5 / 2$ and $3 \mathrm{~d} 3 / 2$, respectively, which could be assigned to $\mathrm{Br}$ in the monovalent oxidation state. $^{25}$

The optical absorption properties of $\mathrm{BiOBr}$ and $\mathrm{Bi} / \mathrm{BiOBr}$ were investigated by UV-vis spectroscopy (Fig. 4). The typical visible light absorption was observed in each sample, which mainly corresponded to the intrinsic narrow band-gap energy of BiOBr. ${ }^{26}$ It can be observed that the absorption ability of the Bi/ $\mathrm{BiOBr}$ heterostructure has been greatly enhanced compared to that of bare BiOBr, which can be attributed to SPR as a result of the appearance of metallic $\mathrm{Bi}^{2}{ }^{27-29}$ The colour change from milky white for the BiOBr sample to dark grey for the $\mathrm{Bi} / \mathrm{BiOBr}$ sample also suggests that the $\mathrm{Bi} / \mathrm{BiOBr}$ sample could utilize more solar light. Furthermore, the specific surface area and porous nature of $\mathrm{Bi} / \mathrm{BiOBr}$ and $\mathrm{BiOBr}$ were measured. As shown in Fig. $\mathrm{S} 2 \dagger$ and Table 1 , the $\mathrm{BET}$ specific surface area of $\mathrm{Bi} / \mathrm{BiOBr}$ is
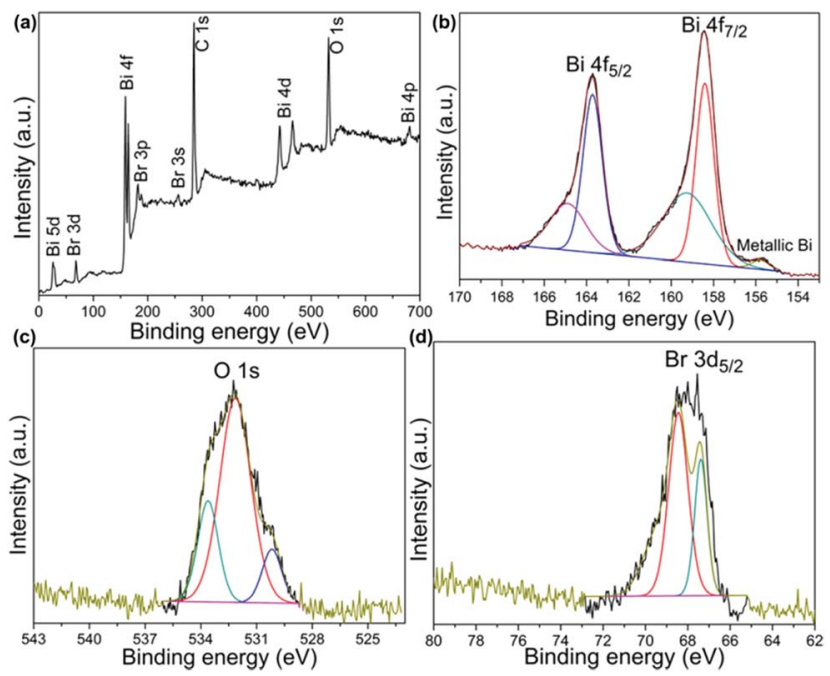

Fig. 3 XPS spectra of the Bi/BiOBr nanoflowers: (a) survey; (b) Bi 4f; (c) $\mathrm{O}$ 1s; (d) Br 3d.

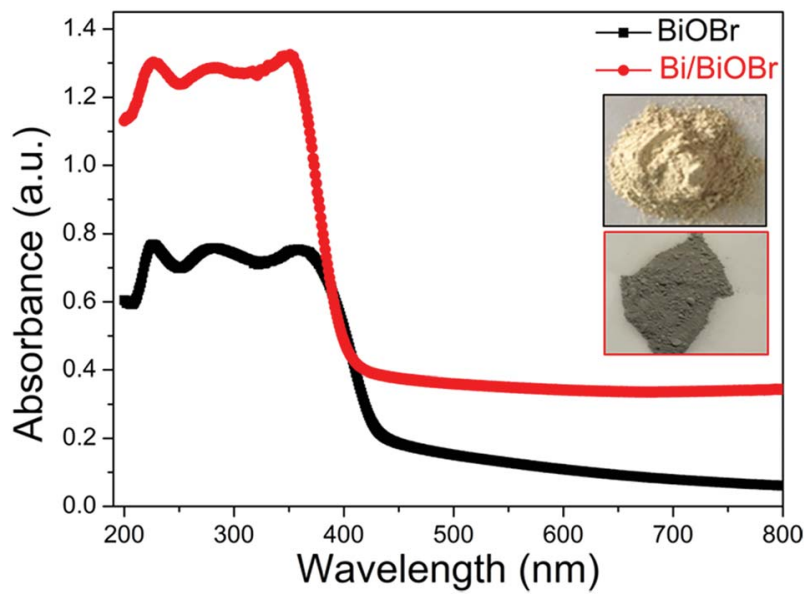

Fig. 4 UV-vis solid absorption spectra of $\mathrm{Bi} / \mathrm{BiOBr}$ and $\mathrm{BiOBr}$ samples.

approximately $20.62 \mathrm{~m}^{2} \mathrm{~g}^{-1}$ and was 3 times higher than that of $\operatorname{BiOBr}\left(6.69 \mathrm{~m}^{2} \mathrm{~g}^{-1}\right)$, which is favourable for its use as a photocatalyst.

When this preparation method was extended to BiOCl and BiOI, similar morphology was obtained (Fig. S3 and S4 $\dagger$ ), but the in situ decoration of $\mathrm{Bi}$ on $\mathrm{BiOCl}$ and BiOI failed (Fig. S5 $\dagger$ ). Therefore, it is important to understand how the Bi selectively forms in the $\mathrm{BiOBr}$ system. Considering the stability constants of BiOCl $\left(K_{\mathrm{sp}}=1.8 \times 10^{-31}\right)$, $\mathrm{BiOBr}\left(K_{\mathrm{sp}}=3 \times 10^{-7}\right)$ and BiOI $\left(K_{\mathrm{sp}}=3 \times 10^{-17}\right)$, the $\mathrm{Bi}^{3+}$ ions will more easily dissociate in $\mathrm{BiOBr}$, which benefits the reduction of $\mathrm{Bi}^{3+}$ to $\mathrm{Bi}^{30-32}$ Moreover, DEG plays a critical role in the nucleation or growth control of $\operatorname{BiOX}(\mathrm{X}=\mathrm{Cl}, \mathrm{Br}$ and $\mathrm{I})$ hierarchical microflowers in this experiment. In detail, the roles of DEG can be divided into three parts: (1) solvent; (2) "soft" template and/or capping agent; ${ }^{33,34}$ and (3) reducing agent. ${ }^{35,36}$ DEG can coordinate with $\mathrm{Bi}^{3+}$ to form a homogeneous precursor prior to decomposition, which is similar to ZnSe microflowers and $\mathrm{Ni}_{7} \mathrm{~S}_{6}$ microflowers. Owing to the large stability constant, $\mathrm{Bi}^{3+}$ was partially reduced to $\mathrm{Bi}$ by DEG in the case of the BiOBr system.

Recently, much attention has been paid to antibiotic photodegradation under visible light irradiation. In the present work, we selected TC as the target compound to evaluate the photodegradation activity of the Bi/BiOBr heterostructure. Fig. 5 shows the relationship of TC concentration variations with irradiation times for $\mathrm{Bi} / \mathrm{BiOBr}$ and $\mathrm{BiOBr}$ samples. The photodegradation of antibiotics by the $\mathrm{Bi} / \mathrm{BiOBr}$ nanomaterial is the combined effect of adsorption, photolysis and photocatalysis. ${ }^{37}$ Control experiments of the adsorption and photolysis effect demonstrate negligible photoactivities, suggesting that the reaction is mainly driven by a photocatalytic process. $\mathrm{Bi} / \mathrm{BiOBr}$

Table 1 Specific surface area and pore structure of the samples

\begin{tabular}{lcll}
\hline Sample & $\begin{array}{l}\text { Specific surface } \\
\text { area }\left(\mathrm{m}^{2} \mathrm{~g}^{-1}\right)\end{array}$ & $\begin{array}{l}\text { Pore size } \\
(\mathrm{nm})\end{array}$ & $\begin{array}{l}\text { Pore volume } \\
\left(\mathrm{cm}^{3} \mathrm{~g}^{-1}\right)\end{array}$ \\
\hline $\mathrm{BiOBr}$ & 6.69 & 22.04 & 0.03 \\
$\mathrm{Bi} / \mathrm{BiOBr}$ & 20.62 & 8.53 & 0.05
\end{tabular}




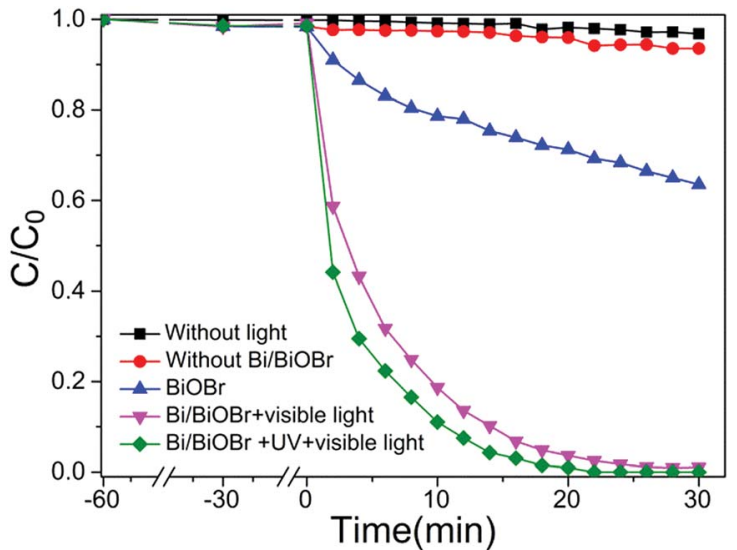

Fig. 5 Photocatalytic activities for degradation of TC solution under visible-light irradiation at room temperature in the presence of $\mathrm{Bi}$ / $\mathrm{BiOBr}$ and $\mathrm{BiOBr}$ samples.

exhibits an extraordinarily high antibiotic photodegradation efficiency (almost 100\% TC removed within $20 \mathrm{~min}$ ), which is obviously higher than that of the $\mathrm{BiOBr}$ photocatalyst $(30 \% \mathrm{TC}$ removed within $40 \mathrm{~min}$ ). In addition, the effect of the filter cutoff $(\lambda \leq 400 \mathrm{~nm})$ on the antibiotic photodegradation was investigated, and high antibiotic photodegradation efficiency under UV and visible light irradiation was observed. For comparison, the photocatalytic performance of the BiOI nanomaterial was also investigated, and the decomposition of TC proceeded much slower than for the $\mathrm{Bi} / \mathrm{BiOBr}$ sample even though they have very similar morphologies (Fig. S6†). The photodegradation of DOX and CIP antibiotics was also studied. DOX and CIP could be completely degraded after $20 \mathrm{~min}$ of irradiation (Fig. S7†). Meanwhile, photodegradation with the BiOBr sample was relatively slow, i.e., $28 \%$ of DOX or CIP can be removed after $40 \mathrm{~min}$ of irradiation. In summary, compared to previously reported literature, the $\mathrm{Bi} / \mathrm{BiOBr}$ microflowers exhibited a superior photodegradation antibiotic ability. ${ }^{38}$

It is common knowledge that several kinds of antibiotics are present in real pollutant wastewater. Therefore, efficient and simultaneous removal of multiple antibiotics is urgently needed. The simultaneous photodegradation of two-component/threecomponent antibiotics was studied. In the case of twocomponent antibiotic photodegradation (Fig. $\mathrm{S} 8 \dagger$ ), $\mathrm{Bi} / \mathrm{BiOBr}$ nanoflowers exhibited much higher photocatalytic activities than $\mathrm{BiOBr}$ nanoplates. Emphatically, the excellent photodegradation performance was also retained in three-component antibiotic degradation experiments. Fig. 6 shows the relationship of DOX, CIP and TC concentration variations with irradiation time for $\mathrm{Bi} / \mathrm{BiOBr}$ and $\mathrm{BiOBr}$ sample nanomaterials. It was clearly observed that for the TC/CIP/DOX three-component antibiotics, the $\mathrm{Bi} / \mathrm{BiOBr}$ photocatalyst also displayed a significantly higher photocatalytic activity than the BiOBr photocatalyst. After $20 \mathrm{~min}$ of irradiation, $99 \%$ of CIP or DOX and $88 \%$ of TC can be degraded in the presence of the $\mathrm{Bi} / \mathrm{BiOBr}$ photocatalyst. Additionally, all TC/ CIP/DOX mixed antibiotics were decomposed within 30 minutes, and the degradation rate of the multiple-component antibiotics was slower than that of the single-antibiotic solution.

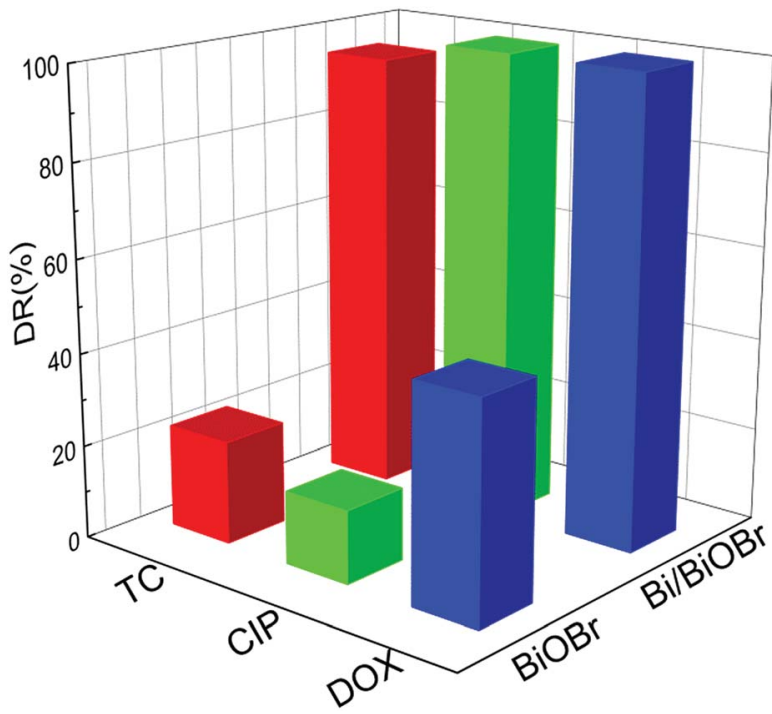

Fig. 6 The photocatalytic degradation of ternary mixed antibiotic TC$\mathrm{DOX}-\mathrm{CIP}$ solution with 20 min irradiation time for $\mathrm{Bi} / \mathrm{BiOBr}$ and $\mathrm{BiOBr}$ samples.

To check the stability of the photocatalytic process, we carried out a photocatalytic experiment under prolonged light illumination. We can find in Fig. 7 that the photocatalytic activity can be retained without a noticeable decrease after five cycles, demonstrating the excellent stability of the $\mathrm{Bi} / \mathrm{BiOBr}$ nanomaterial. Additionally, the SEM and XRD patterns of $\mathrm{Bi}$ / BiOBr were investigated for 5 reusability runs in TC photodegradation. There is no obvious change in the photocatalytic reaction. Moreover, XPS results display the peak at about $155.7 \mathrm{eV}$ that is assigned to metallic $\mathrm{Bi}$, which further confirms the existence of metallic Bi after photocatalytic reaction. These results suggest that our $\mathrm{Bi} / \mathrm{BiOBr}$ nanomaterial is an efficient and stable visible light photocatalyst.
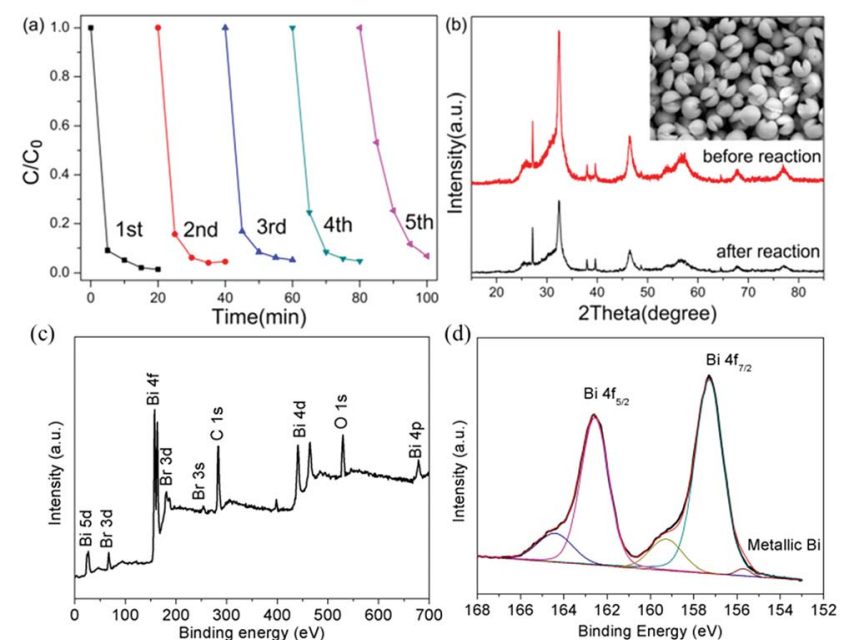

Fig. 7 (a) Reusability of $\mathrm{Bi} / \mathrm{BiOBr}$ microflowers for photodegradation of TC; (b) XRD pattern and SEM result before and after 5 reusability tests; XPS spectra of the $\mathrm{Bi} / \mathrm{BiOBr}$ after 5 reusability tests: (c) survey; (d) Bi $4 f$. 


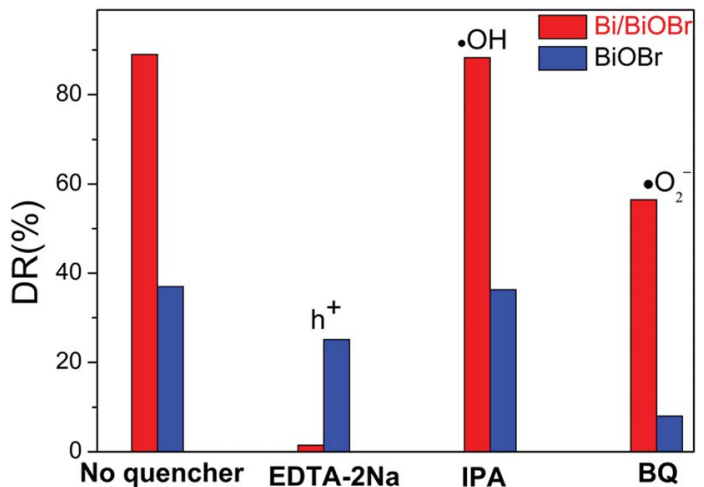

Fig. 8 Trapping experiment of active species during the photocatalytic reaction with visible light irradiation: EDTA-2Na as a scavenger for holes $\left(h^{+}\right)$; IPA (iso-propanol) as a scavenger for hydroxyl radicals $(\cdot \mathrm{OH})$; $\mathrm{BQ}(1,4$-benzoquinone) as a scavenger for superoxide radicals $\left(\cdot \mathrm{O}_{2}^{-}\right)$

In order to confirm the main active radical species and the potential reaction mechanism for superior photodegradability of the $\mathrm{Bi} / \mathrm{BiOBr}$ nanomaterial, trapping experiments of active species were performed via photocatalytic reaction (Fig. 8). The degradation rate (DR) decreased in the presence of superoxide radical scavenger BQ and hole scavenger EDTA-2Na, revealing that $\cdot \mathrm{O}_{2}{ }^{-}$and $\mathrm{h}^{+}$are both active species for TC degradation, and the holes $\mathrm{h}^{+}$play the dominant role in the $\mathrm{Bi} / \mathrm{BiOBr}$ system, while $\cdot \mathrm{O}_{2}{ }^{-}$plays a stronger role than $\mathrm{h}^{+}$in the presence of the $\mathrm{BiOBr}$ sample. However, the $\mathrm{CB}$ of $\mathrm{BiOBr}$ is positive relative to the potential of $\mathrm{O}_{2} / \cdot \mathrm{O}_{2}{ }^{-}(-0.33 \mathrm{~V}$ versus $\mathrm{NHE})$, so $\mathrm{CB}$ electrons are unable to easily produce $\cdot \mathrm{O}_{2}{ }^{-} \cdot{ }^{39}$ However, $\mathrm{BiOBr}$ reacts with $\mathrm{O}_{2}$ under irradiation, and a similar phenomenon was reported in previous work. ${ }^{40}$ Because the electrons excited into the $\mathrm{CB}$ of BiOBr would induce a Fermi level up-shift and benefit the reduction of $\mathrm{O}_{2}$, the decoration of $\mathrm{Bi}$ significantly reduces the process of generating $\cdot \mathrm{O}_{2}{ }^{-}$, which demonstrates that $\mathrm{Bi}$ as a cocatalyst is inert to the reaction of $\mathrm{O}_{2} / \cdot \mathrm{O}_{2}{ }^{-}$. Due to the position difference between BiOBr's CB $(0.15 \mathrm{eV})$ and the Bi Fermi level

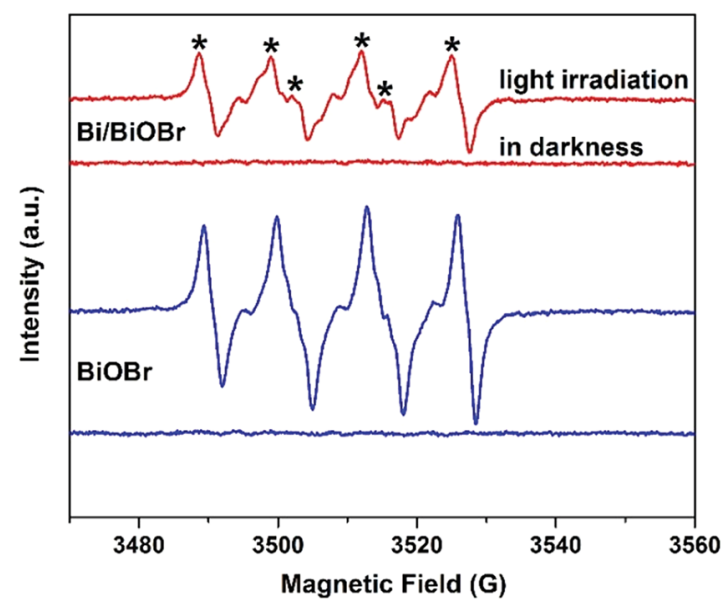

Fig. 9 ESR spectra of the as-synthesized $\mathrm{Bi} / \mathrm{BiOBr}$ and $\mathrm{BiOBr}$ samples for $\mathrm{DMPO}-\cdot \mathrm{O}_{2}^{-}$.
$(-0.17 \mathrm{eV})$, photogenerated electrons migrate easily from $\mathrm{Bi}$ to the $\mathrm{CB}$ of $\mathrm{BiOBr}$; therefore, the heterostructure favours the separation process of charges and promotes the activity of $\mathrm{h}^{+}$for degradation. The above deduction can be well supported by the ESR analysis (Fig. 9). Obvious DMPO-· $\mathrm{O}_{2}{ }^{-}$characteristic peaks appeared after light irradiation, which further indicates the generation of $\cdot \mathrm{O}_{2}{ }^{-}$during the degradation of TC, but the signal density was weaker in the presence of $\mathrm{Bi} / \mathrm{BiOBr}$ because of the decreased amount of $\cdot \mathrm{O}_{2}{ }^{-}$, which agrees well with the trapping experiment. The joint action of $\cdot \mathrm{O}_{2}{ }^{-}$and $\mathrm{h}^{+}$leads to the enhanced photodegradation performance of $\mathrm{Bi} / \mathrm{BiOBr}$.

\section{Conclusions}

In summary, a novel visible-light-responsive photocatalytic $\mathrm{Bi}$ / $\mathrm{BiOBr}$ heterostructure has been prepared via a simple in situ solvothermal synthetic route. The $\mathrm{Bi} / \mathrm{BiOBr}$ photocatalyst shows excellent visible light photocatalytic properties for antibiotic degradation, compared to bare BiOBr. In two-component/threecomponent antibiotic solutions, almost $100 \%$ of the antibiotics were degraded within $30 \mathrm{~min}$ of irradiation. This high photocatalytic activity originates from its hierarchical microflowers. And the photocatalytic activity and structure were retained after 5 cycles. This work demonstrated a powerful strategy for the in situ reduction and confined growth of well-defined 3D heterostructures, and this promising performance shows great potential for these materials as photocatalysts for the visible light removal of multiple antibiotics in practical application.

\section{Conflicts of interest}

There are no conflicts to declare.

\section{Acknowledgements}

We acknowledge the National Key Research and China Program (2016YFB0701100) and NSFC (21671083, 51525101, 51601032 and 51402047).

\section{Notes and references}

1 A. Fujishima and K. Honda, Nature, 1972, 37, 238.

2 S. Y. Wang, Y. Y. Gao, S. Miao, T. F. Liu, L. C. Mu, R. G. Li, F. T. Fan and C. Li, J. Am. Chem. Soc., 2017, 139, 11771.

3 M. Yan, Y. Q. Hua, F. F. Zhu, W. Gu, J. H. Jiang, H. Q. Shen and W. D. Shi, Appl. Catal., B, 2017, 202, 518.

4 A. Han, H. W. Zhang, D. Lu, J. L. Sun, G. K. Chuah and S. Jaenicke, J. Hazard. Mater., 2018, 341, 83.

5 E. M. Ferguson, M. Allinson, G. Allinson, S. E. Swearer, K. L. Hassell and P. P. Bay, Water Res., 2012, 47, 1604.

6 J. Li, Y. Yu and L. Z. Zhang, Nanoscale, 2014, 6, 8473.

7 W. Q. Fan, C. F. Li, H. Y. Bai, Y. Y. Zhao, B. F. Luo, Y. J. Li, Y. L. Ge, W. D. Shi and H. P. Li, J. Mater. Chem. A, 2017, 5, 4894.

8 C. L. Yu, C. F. Fan, X. J. Meng, K. Yang, F. F. Cao and X. Lin, React. Kinet., Mech. Catal., 2011, 103, 141. 
9 C. L. Yu, F. F. Cao, G. Li, R. F. Wei, J. C. Cui, R. C. Jin, Q. Z. Fan and C. Y. Wang, Sep. Purif. Technol., 2013, 120, 110.

10 C. L. Yu, J. C. Cui, C. F. Fan, H. R. Wen and S. J. Hu, Mater. Sci. Eng., B, 2010, 166, 213.

11 Y. Yu, C. Y. Cao, H. Liu, P. Li, F. F. Wei, Y. Jiang and W. G. Song, J. Mater. Chem. A, 2014, 2, 1677.

12 Z. W. Zhao, W. D. Zhang, Y. J. Sun, J. Y. Yu, Y. X. Zhang, H. Wang, F. Dong and Z. B. Wu, J. Phys. Chem. C, 2016, 120, 11889.

13 X. M. Zhang, G. B. Ji, Y. S. Liu, X. G. Zhou, Y. Zhu, D. N. Shi, P. Zhang, X. Z. Cao and B. Y. Wang, Phys. Chem. Chem. Phys., 2015, 17, 8078.

14 C. R. Zheng, C. B. Cao and Z. Ali, Phys. Chem. Chem. Phys., 2015, 17, 13347.

15 M. C. Gao, D. F. Zhang, X. P. Pu, H. Li, D. D. Lv, B. B. Zhang and X. Shao, Sep. Purif. Technol., 2015, 154, 211.

16 Q. S. Wang, L. X. Song, Y. Teng, J. Xia, L. Zhao and M. M. Ruan, RSC Adv., 2015, 5, 80853.

17 H. C. Ma, M. Zhao, H. M. Xing, Y. H. Fu, X. F. Zhang and X. L. Dong, J. Mater. Sci.: Mater. Electron., 2015, 26, 10002.

18 X. A. Dong, W. D. Zhang, Y. J. Sun, J. Y. Li, W. L. Cen, Z. H. Cui, H. W. Huang and F. Dong, J. Catal., 2018, 357, 41.

19 S. Li, J. J. Cai, Y. L. Liu, M. Q. Gao, F. Cao and G. W. Qin, Sol. Energy Mater. Sol. Cells, 2018, 179, 328.

20 L. Y. Wang, X. X. Xu, S. J. Wu and F. Cao, Catal. Sci. Technol., 2018, 8, 1366.

21 X. A. Dong, W. D. Zhang, Y. J. Sun, J. Y. Li, W. L. Cen, Z. H. Cui, H. W. Huang and F. Dong, J. Catal., 2018, 357, 41.

22 Y. X. Guo, Y. H. Zhang, N. Tian and H. W. Huang, ACS Sustainable Chem. Eng., 2016, 4, 4003.

23 S. Kang, R. C. Pawar, Y. Pyo, V. Khare and C. S. Lee, J. Exp. Nanosci., 2016, 11, 259.

24 S. X. Yu, H. W. Huang, F. Dong, M. Li, N. Tian, T. R. Zhang and Y. H. Zhang, ACS Appl. Mater. Interfaces, 2015, 7, 27925.
25 Z. Q. He, D. Wang, H. Y. Fang, J. M. Chen and S. Song, Nanoscale, 2014, 6, 10540.

26 H. Wang, S. C. Chen, D. Y. Yong, X. D. Zhang, S. Li, W. Shao, X. S. Sun, B. C. Pan and Y. Xie, J. Am. Chem. Soc., 2017, 139, 4737.

27 S. Bai, X. Y. Li, Q. Kong, R. Long, C. M. Wang, J. Jiang and Y. J. Xiong, Adv. Mater., 2015, 27, 3444.

28 C. Y. Wang, Y. J. Zhang, W. K. Wang, D. N. Pei, G. X. Huang, J. J. Chen, X. Zhang and H. Q. Yu, Appl. Catal., B, 2018, 221, 320.

29 H. Li, F. Qin, Z. P. Yang, X. M. Cui, J. F. Wang and L. Z. Zhang, J. Am. Chem. Soc., 2017, 139, 3513.

30 J. A. Dean, Lange's handbook of chemistry, 1985.

31 X. Y. Gao, X. C. Zhang, Y. W. Wang, S. Q. Peng, B. Yue and C. M. Fan, Chem. Eng. J., 2015, 263, 419.

32 K. X. Ren, J. Liu, J. Liang, K. Zhang, X. Zheng, H. D. Luo, Y. B. Huang, P. J. Liu and X. B. Yu, Dalton Trans., 2013, 42, 9706.

33 F. Cao, Y. N. Wang, J. M. Wang, X. Lv, D. Y. Liu, J. Ren, J. Zhou, R. Deng, S. Li and G. W. Qin, New J. Chem., 2018, 42, 3614.

34 F. Cao, R. X. Liu, L. Zhou, S. Y. Song, Y. Q. Lei, W. D. Shi, F. Y. Zhao and H. J. Zhang, J. Mater. Chem., 2010, 20, 1078.

35 C. Feldmann and H. O. Jungk, Angew. Chem., Int. Ed., 2001, 40, 359.

36 W. J. Xu, F. L. Lyu, Y. C. Bai, A. Q. Gao, J. Feng, Z. X. Cai and Y. D. Yin, Nano Energy, 2018, 43, 110.

37 L. Q. Ye, Y. R. Su, X. L. Jin, H. Q. Xie and C. Zhang, Environ. Sci.: Nano, 2014, 1, 90.

38 H. F. Cheng, B. B. Huang and Y. Dai, Nanoscale, 2014, 6, 2009.

39 Y. Z. Hong, C. S. Li, B. X. Yin, D. Li, Z. Y. Zhang, B. D. Mao, W. Q. Fan, W. Gu and W. D. Shi, Chem. Eng. J., 2018, 338, 137. 40 H. W. Huang, X. Han, X. W. Li, S. C. Wang, P. K. Chu and Y. H. Zhang, ACS Appl. Mater. Interfaces, 2015, 7, 482. 